\title{
Brain oscillatory activity during tactile stimulation correlates with cortical thickness of intact areas and predicts outcome in comatose patients
}

\author{
Galina Portnova ${ }^{1}$, Irina Girzhova ${ }^{2}$, Daria Filatova ${ }^{2}$, Vitaliy Podlepich ${ }^{3}$, Alina Tetereva ${ }^{1}$, Olga \\ Martynova ${ }^{1}$ \\ ${ }^{1}$ Human High Nervous Activity Laboratory, Institute of Higher Nervous Activity and \\ Neurophysiology of the Russian Academy of Science \\ ${ }^{2}$ Faculty of Fundamental Medicine, Lomonosov Moscow State University \\ ${ }^{3}$ Federal State Autonomous Institution N. N. Burdenko National Medical Research Center of \\ Neurosurgery of the Ministry of Health of the Russian Federation, Moscow, Russian Federation
}

\begin{abstract}
This study reports a correlation between EEG and structural brain changes in patients after severe traumatic brain injury in a coma. The novelty of our approach was based on the combination of structural visualization (MRI) and functional neuroimaging (EEG) during tactile stimulation. The structural morphometry indicated a decrease of whole-brain cortical thickness, the gray-matter volume of the cortex, and subcortical structures in comatose patients compared to healthy subjects. In resting-state EEG, coma patients had significantly higher power of the slow-wave activity of $2-6 \mathrm{~Hz}$ and significantly less power of the alpha and beta rhythm. Importantly, coma patients showed a significant decrease of theta-rhythm power in tactile stimulation compared to the resting state, and this EEG pattern was not found in the control group. The decrease of the theta-rhythm power significantly correlated with the better outcome from a coma. Spectral changes in EEG in response to tactile stimuli showed no association with brain morphometric measures in healthy controls. In patients, decreasing theta-rhythm power correlated positively with the volume of whole-brain gray matter, right putamen, and insula; and negatively with the volume of damaged brain tissue. Increasing beta-rhythm power, specific tactile EEG response for a healthy brain, correlated with the cortical thickness of the somatosensory Paracentral and Precentral area. The observed decrease of gray-matter volume indicates brain atrophy in coma patients, which could be associated with neurodegeneration induced by injury. Our results also demonstrate that slow-wave desynchronization, as a nonspecific response to tactile stimulation, can serve as a sensitive index of morphometric changes after brain injury and coma outcome.
\end{abstract}

Keywords: coma, unconsiousness, EEG, MRI, Freesurfer, TBI. 


\section{EEG in response to tactile stimulation in a coma}

\section{Introduction}

The relationship between brain damage and neurological mechanisms supporting recovery after severe brain injury remains largely unknown [1]. The connection between behaviorally defined clinical entities and the underlying brain damage was also poorly investigated. MRI studies demonstrated an association between a patient's quantitative clinical measures of behavioral responsiveness and arousal, and the degree of tissue atrophy within the cortex, thalamus, basal ganglia, and basal forebrain [2-4]. Peaks of maximal atrophy occurred in bilateral anterodorsal thalami, anterior dorsomedial caudate nuclei, and anterior hippocampus. At the same time, patients after severe TBI without visible neuroradiological lesions had the volume reduction of the hippocampus, fornix, and other structures [5]. According to the consciousness framework of Crick and Koch (2003), there are brain networks necessary to provide the consciousness processes, including sensory, semantic, and motor neural chains [6]. These neural coalitions could be described as a subset of cortical structures, including sensorimotor areas, prefrontal, posteromedial cortex cingulate, and parietal regions, as well as the relevant thalamocortical loops, that capable of integrating the supervision, limited-capacity, and re-entry properties of conscious sensory processing [7]. The impairment of the networks, including frontoparietal, thalamocortical network encompassing the polymodal associative cortices induced the impairment of consciousness [8-13].

However, data on the association between recovery after severe TBI and the level of brain damage are rather diverse. The better recovery was accompanied by a functional restoration of the frontoparietal network [10] and some of its thalamocortical connections [13]. Prediction of recovery from coma relies on the comprehensive assessment of the ascending reticular activating system located in the posterosuperior part of the brainstem and structures encompassing thalamus, basal forebrain, and frontoparietal association cortices [1,11]. Studies on traumatic coma patients with conventional MRI showed that lesions of the pons, midbrain, and basal ganglia were predictive of poor outcomes, especially when they were bilateral [17]. The length of coma and memory loss was predicted by the callosal volume reduction, which could be considered a marker of axonal loss [18]. The increased frequency of deep supratentorial and stem lesions was correlated with an increased incidence of coma and unfavorable outcomes, while cortical and subcortical white matter lesions were associated with better neurological status and prognosis. The involvement of corpus callosum, subcortex, and stem was associated with a coma and increased incidence of unfavorable outcomes [19]. According to R. Firsching et al. [20], establishing the exact location of the primary and secondary brainstem lesions is crucial in predicting severe TBI outcomes.

Functional neuroimaging such as electroencephalography (EEG) also demonstrated variable abnormal activity in a coma after traumatic brain injury. Mecarelli et al. showed that the background activity progressively decreased in amplitude until the eventual attenuation or suppression of the cortical bioelectrical activity [14]. Spindle coma accompanied pontomesencephalic junction lesion when epileptiform activity and sleep spindles appear along with functional preservation of the rest EEG patterns [15]. The beta coma pattern was most commonly induced by an acute brainstem lesion, while alpha coma patterns were detected in patients with pontomesencephalic hemorrhage or other acute encephalopathies [16].

It should be noted that EEG patterns are not specific for a single etiology, and the general recovery prognosis depends on several factors, including the severity of the damage, type of injury, neurologic findings, and patient age [21]. However, there are individual EEG indices that may help evaluate outcome prognosis. For example, alpha coma pattern and low-voltage, slow, nonreactive patterns were generally considered as a biomarker for poor outcomes [14, 22]; 


\section{EEG in response to tactile stimulation in a coma}

spindle coma was associated with good outcome [15]. Patients with bilateral independent periodic lateralized epileptiform discharges had a higher mortality rate compared to patients with unilateral [21].

The prediction of coma outcome based only on MRI or EEG data is still problematic. The previous studies demonstrated that the combined clinical and neuroimaging approach gives a much better prediction [23]. Further, reactivity to external responses is more informative than underlying EEG rhythms on their own. Responses to auditory, noxious, tactile, and visual stimuli must be examined, looking for changes in the underlying pattern of steady-state EEG [24]. In particular, EEG in response to tactile stimulation in correlation with brain morphometry measures could be one of the effective methods to predict the recovery from coma. Early, it has been suggested that the absence of response to tactile stimulation was a more useful predictor of poor outcomes than most clinical variables [12]. This assumption is also supported by the data indicating the activating effect of tactile stimulation on human consciousness [25-29, 33].

Additionally, tactile stimuli may cause a significant impact on the human emotional conditions via the C-tactile (CT) fibers responding vigorously to pleasant types of tactile experiences and supporting the system of the affective-motivational dimension of touch [30]. Functional magnetic resonance imaging (fMRI) studies have shown that preferential CT fiber stimulation activates the posterior insula cortex [32]. Several brain regions, beyond the insula, are also involved in the perception of tactile: the right posterior superior temporal sulcus, medial prefrontal cortex, and cingulate cortex [31]. Studies in adult neuronopathy patients who lack A $\beta$ fibers have shown that pure CT stimulation activates the insular cortex but not the somatosensory regions [28].

In the present study, we hypothesized that tactile stimulation could have a restorative impact on unconscious patients after severe TBI affecting both $\mathrm{A} \beta$ and CT fibers, which have different cortical projections. CT stimulation is a perspective approach in rehabilitation of comatose patients, as it affects the emotional system [30] and, as a result, should have a more significant impact on patients' recovery of consciousness. The evoked oscillatory response to tactile stimulation could also serve as a potential biomarker, predicting coma outcome. We compared oscillatory brain activity during resting state and in response to tactile stimulation in healthy controls and TBI patients. Then we investigated the correlation of EEG indices with brain morphometry data and coma outcome in TBI patients. The structural MRI also supported the lack of damage to brain areas connected with tactile afferentation.

\section{Methods}

\section{Participants}

Ten patients ( $37.8 \pm 29.8$ y.o.( $(\mathrm{M} \pm 2 \sigma), 8$ males, 2 females) after severe TBI participated in the study via inpatient observation in N.N. Burdenko National Scientific and Practical Center for Neurosurgery from 2017 to 2018. The average length of total hospital stay after TBI (including intensive care unit) was $22.05 \pm 57.58$ days. The causes of severe TBI are the following: car accident, fall from a height, beating. The outcomes are the following: transfer to another hospital, transfer to a rehabilitation center, recovery, death (Table 1). The average of the Glasgow Coma Scale at the beginning of the hospital treatment was 5,9; the average outcome (Glasgow Outcome Scale extended) was 4,6 (see Table 1). 


\section{EEG in response to tactile stimulation in a coma}

The control group consisted of 15 healthy volunteers (29.6 \pm 3.5 years old). Exclusion criteria were pregnancy, psychiatric or neurological disorders, and the use of any interfering medications and recreational drugs. The healthy participants reported not having any current or past neurological or psychological disorders. The participants were informed about the experimental procedure. The study was conducted in accordance with the Helsinki Declaration, and the study protocol was approved by the Ethics Department of the Institute of Higher Nervous Activity and Neurophysiology of RAS (project identification code AAAA-A17117092040004-0; protocol \#6 from 19/12/2019) and in N.N. Burdenko National Scientific and Practical Center for Neurosurgery. All participants or their legal representative provided written informed consent.

\section{Stimuli}

Four types of tactile were used:

1) Soft brush (60 mm, squirrel hair), slow stroking $(2-3 \mathrm{~cm} / \mathrm{s})$;

2) Soft brush (60 mm, squirrel hair), fast stroking $(12-15 \mathrm{~cm} / \mathrm{s})$;

3) Hard brush (60 mm, natural bristle), slow stroking $(2-3 \mathrm{~cm} / \mathrm{s})$;

4) Hard brush $(60 \mathrm{~mm}$, natural bristle), fast stroking $(12-15 \mathrm{~cm} / \mathrm{s})$.

\section{Procedure}

The stimulation was applied to the hairy part of a left forearm. Each stimulus was presented for $10 \mathrm{sec}$ in a randomized order with an interval of 2-10 sec. Each stimulus was presented 10 times.

A stimuli delivery sequence was composed in Presentation (Neurobehavioral Systems), that visualized stimuli type and presentation speed via an animation. An operator was trained to deliver stimulation according to the animation speed.

\section{Subjective assessment of stimuli}

The healthy controls reported about the stimuli in 5 aspects: painfulness, pleasantness, ticklishness, speed, and intensity.

\section{EEG registration}

EEG was acquired using a 19-channel EEG amplifier Encephalan with the recording of polygraphic channels (Poly4, Medicom MTD, Taganrog, Russian Federation). The sampling rate was $250 \mathrm{~Hz}$. The amplifier bandpass filter was nominally set to $0.05-70 \mathrm{~Hz}$. AgCl electrodes (Fp1, Fp2, F7, F3, Fz, F4, F8, T3, C3, Cz, C4, T4, T5, P3, Pz, P4, T6, O1, and O2) were placed according to the International 10-20 system. The electrodes placed on the left and right mastoids served as joint references under unipolar montage. The vertical EOG was recorded with $\mathrm{AgCl}$ cup electrodes placed $1 \mathrm{~cm}$ above and below the left eye, and the horizontal EOG was acquired by electrodes placed $1 \mathrm{~cm}$ lateral from the outer canthi of both eyes. The electrode impedances were kept below $10 \mathrm{k} \Omega$.

\section{EEG data analysis}

Fast Fourier transform was used to analyze power spectral density (PSD). The PSD was estimated for every $300 \pm 7.8 \mathrm{~s}$ intervals. The resulting normalized spectra were integrated over intervals of unit width in the range of interest $(2-3 \mathrm{~Hz}, 3-4 \mathrm{~Hz}, \ldots 19-20 \mathrm{~Hz})$ for all conditions (stimulation and the resting state). 


\section{EEG in response to tactile stimulation in a coma}

\section{MRI acquisition}

The MRI of the control group was obtained by a 3T scanner (Magnetom Verio, Siemens, Germany) at the National Research Center Kurchatov Institute (Moscow, RF). MRI of patients after TBI was obtained by 1,5 T scanner (Signa Horizon, General Electric, USA) at the N. N. Burdenko National Medical Research Center of Neurosurgery (Moscow, RF).

For each subject, a sagittal high-resolution T1-weighted rapid gradient-echo (anatomical) image was acquired using a T1 MP-RAGE sequence: TR $1470 \mathrm{~ms}$, TE $1.76 \mathrm{~ms}$, FA 9, 176 slices with a slice thickness of $1 \mathrm{~mm}$ and a slice gap of $0.5 \mathrm{~mm}$; and a field of view $320 \mathrm{~mm}$ with a matrix size of $320 \times 320$.

\section{Structural morphometry}

Brain morphometry was analyzed in both the control group and coma patients. Each image was processed with a FreeSurfer standard pipeline «recon-all» with default parameters (http://surfer.nmr.mgh.harvard.edu/, version 6.0). The pipeline included the following steps: the segmentation of the subcortical white and gray matter volumetric structures (including ventricles, amygdala, hippocampus, caudate, putamen) [34,35]; intensity normalization [36]; tessellation of the gray matter and white matter boundary; automated topology correction [37,38]; and surface deformation following intensity gradients to optimally place the gray/white and gray/cerebrospinal fluid borders at the location where the most significant shift in intensity defines the transition to the other tissue class [39-41]. When the cortical model was complete, several deformable procedures were performed including surface inflation [42], registration to a spherical atlas which utilizes individual cortical folding patterns to match cortical geometry across subjects [43], parcellation of the cerebral cortex into units based on the gyral and sulcal structure $[44,45]$, and creation of a variety of surface-based data including maps of curvature and sulcal depth [41].

The cortical thickness and voxel volume of subcortical structures were extracted using "aparcstats2table" and "asegstats2table" commands for each subject. A cortical parcellation was acquired, according to Desikan-Killiany Atlas [46].

\section{Statistical analysis}

Factorial ANOVAs followed by post-hoc comparison (Bonferroni, $\mathrm{p}<0.05$ ) determined group effects on EEG metrics. We calculated differences between PSD during tactile stimulation and rest and analyzed averaged values for all tactile stimuli using Wilcoxon analysis by cluster-based permutation test with 500 permutations at each node and Bonferroni correction $(\mathrm{p}<0.05)$ for multiple comparisons (see Figure 3, 4). We analyzed a possible association of the EEG metrics with the ratings of clinical scales and MRI data using Spearman correlation analysis by clusterbased permutation test (Matlab toolbox for BCI) with 500 permutations and Bonferroni correction $(\mathrm{p}<0.05)$. The association between MRI data and the clinical scales was tested using Spearman correlation analysis with Bonferroni correction $(\mathrm{p}<0.05)$.

\section{Results}

\section{Brain atrophy in coma patients}

The structural morphometry showed that trauma-affected brain areas did not overlap with areas associated with tactile perception (see Table1). 


\section{EEG in response to tactile stimulation in a coma}

The whole-brain cortical thickness in comatose patients decreased as compared to healthy subjects (Table 1, Figure 1): $(\mathrm{F}(1,23)=15.241, \mathrm{p}=.00338 ; \mathrm{MS}=10.7)$. The gray-matter volume of cortex and subcortical structures was also significantly lesser in patients, $(\mathrm{F}(1$, 23) $=14,397, \mathrm{p}=, 00196 ; \mathrm{MS}=13.2)($ Table3).
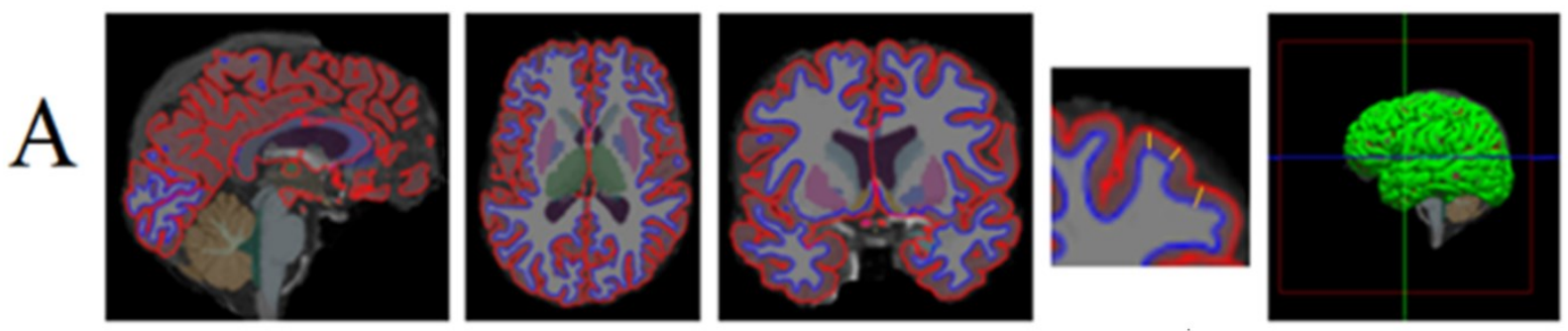

\section{Study group \\ Control group}
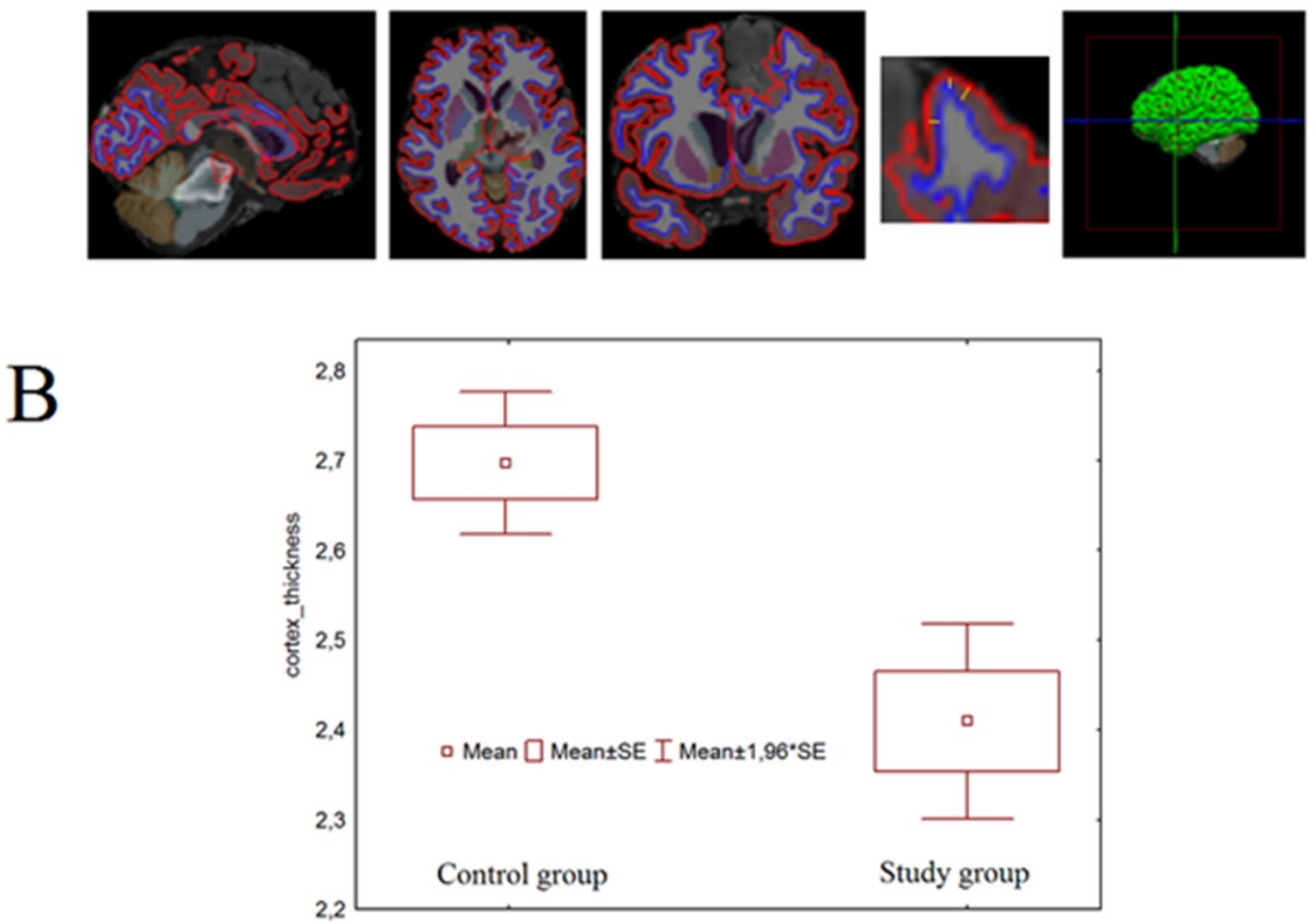

Figure 1. The morphometry results (Freesurfer) showing the difference in the cortical thickness between comatose patients and the control group.

Altered resting-state EEG in coma patients

During rest, comatose patients, compared with the control group, had significantly higher power of the slow-wave activity of $2-6 \mathrm{~Hz}(\mathrm{~F}(1,23)=13.600, \mathrm{p}=.00122 ; \mathrm{MS}=14.2)$ and significantly less power of the alpha $(8-12 \mathrm{~Hz})$ and beta rhythm $(16-20 \mathrm{~Hz})(\mathrm{F}(1,23)=21.967, \mathrm{p}=.00010$; $\mathrm{MS}=16.9$ ). 


\section{EEG in response to tactile stimulation in a coma}

\section{EEG response tactile stimulation}

The tactile stimulation induced the increase of alpha- $(11-13 \mathrm{~Hz})$ and beta-rhythm $(17-20 \mathrm{~Hz})$ PSD in both groups of subjects (Figure 2). According to the morphometry results, the main differences in the cortical thickness were located in the following regions: right insula, anterior cingulate, paracentral, precentral, supramarginal, inferior parietal areas; left insula, anterior and posterior cingulate, precentral, paracentral, supramarginal, middle frontal, parahippocampal, inferior and superior parietal areas.

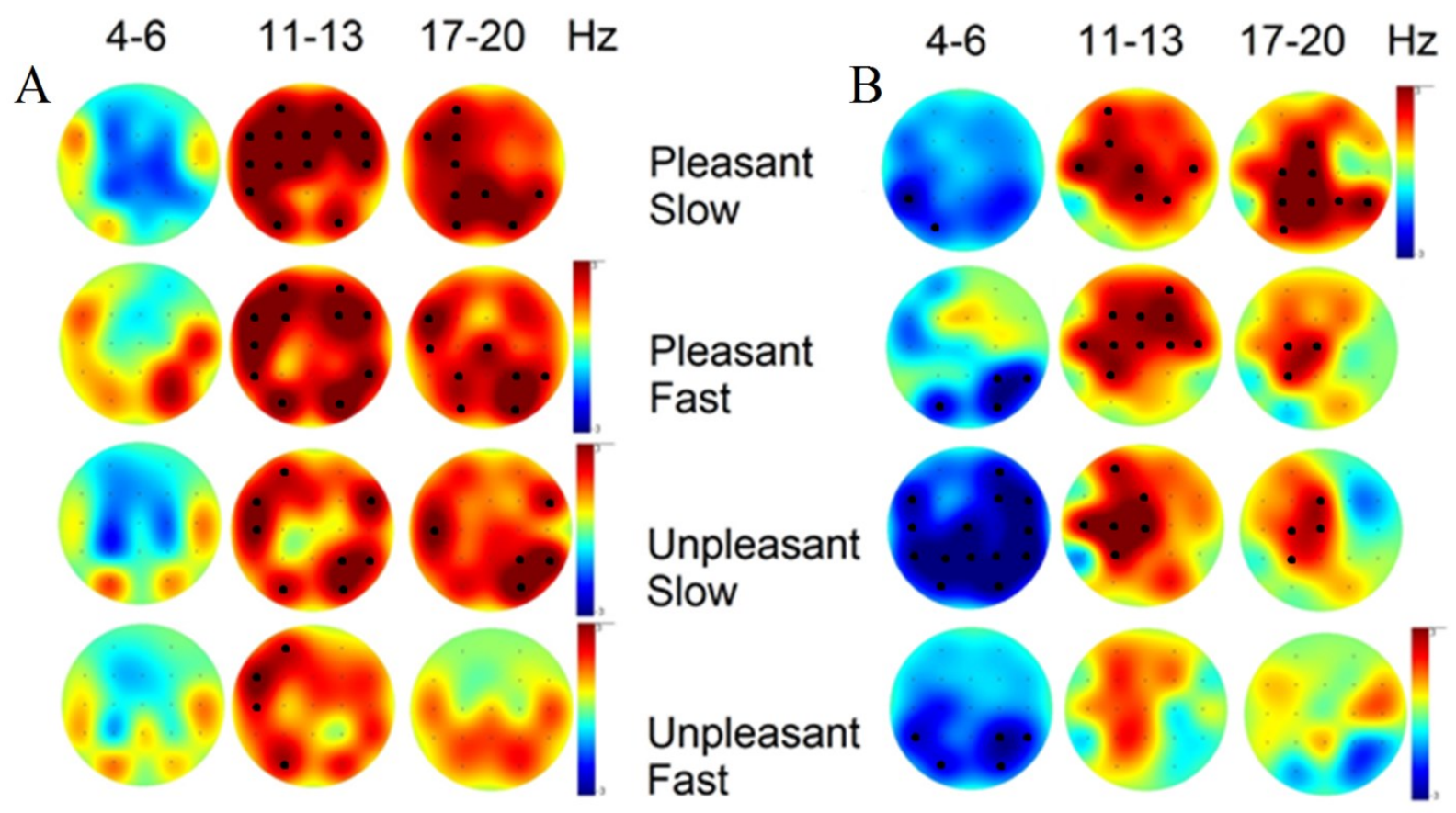

Figure2. The difference in theta-rhythm (4-6 Hz), alpha-rhythm 11-13 Hz, and beta-rhythm PSD between tactile stimulation and the resting states for both groups of subjects. A-control group, $B$ - patients in coma. The bold black dots indicate a significant difference after Bonferroni correction.

Comatose patients showed a significant decrease in theta-rhythm power during tactile stimulation as compared to resting-state EEG. Importantly, this decrease was not observed in the control group (Figure 2) $\mathrm{F}(1,23)=11,694, \mathrm{p}=, 00234, \mathrm{MS}=11.7$ ). The decrease of the thetarhythm power significantly correlated with patients better outcome $(r=0.28, p=0.02)$ (Figure $2 \mathrm{~B}$ )

\section{Correlation of tactile EEG response with morphometric data}

Decrease of theta-rhythm power (the differences between PSD during tactile stimulation and rest) in the occipital and parietal areas correlated with the volume of the whole brain gray matter, right putamen, right insula, and inversely correlated with the volume of damaged brain tissue (Surface holes), see Figure 3. So, the higher was a decrease of the theta-rhythm power in tactile stimulation; the lesser was brain damage and higher volume of gray matter. 
EEG in response to tactile stimulation in a coma

A
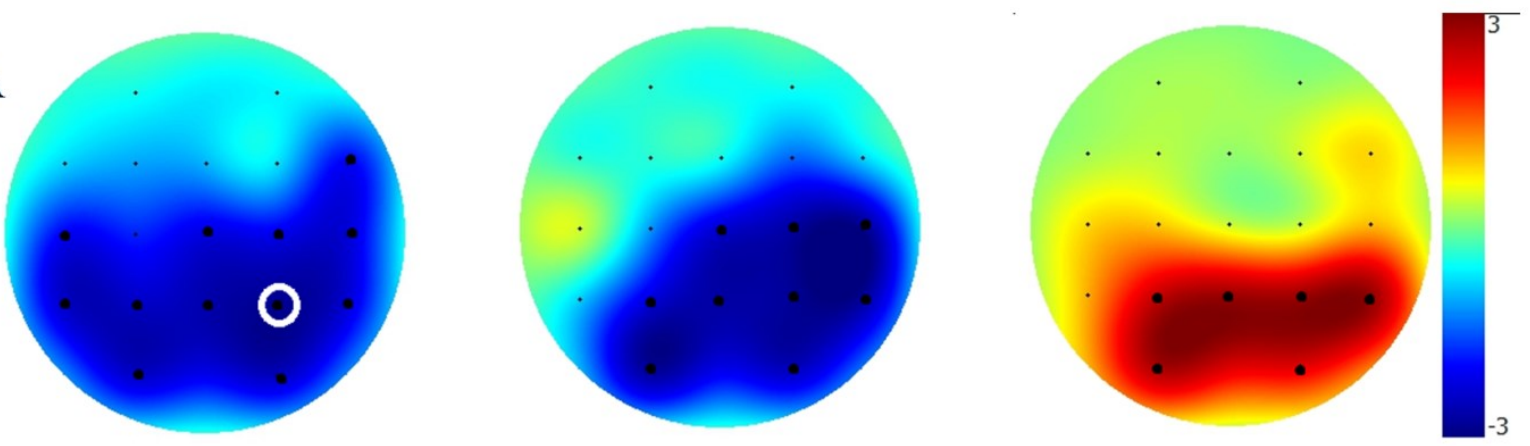

\section{Right Putamen Gray matter volume Surface Holes}

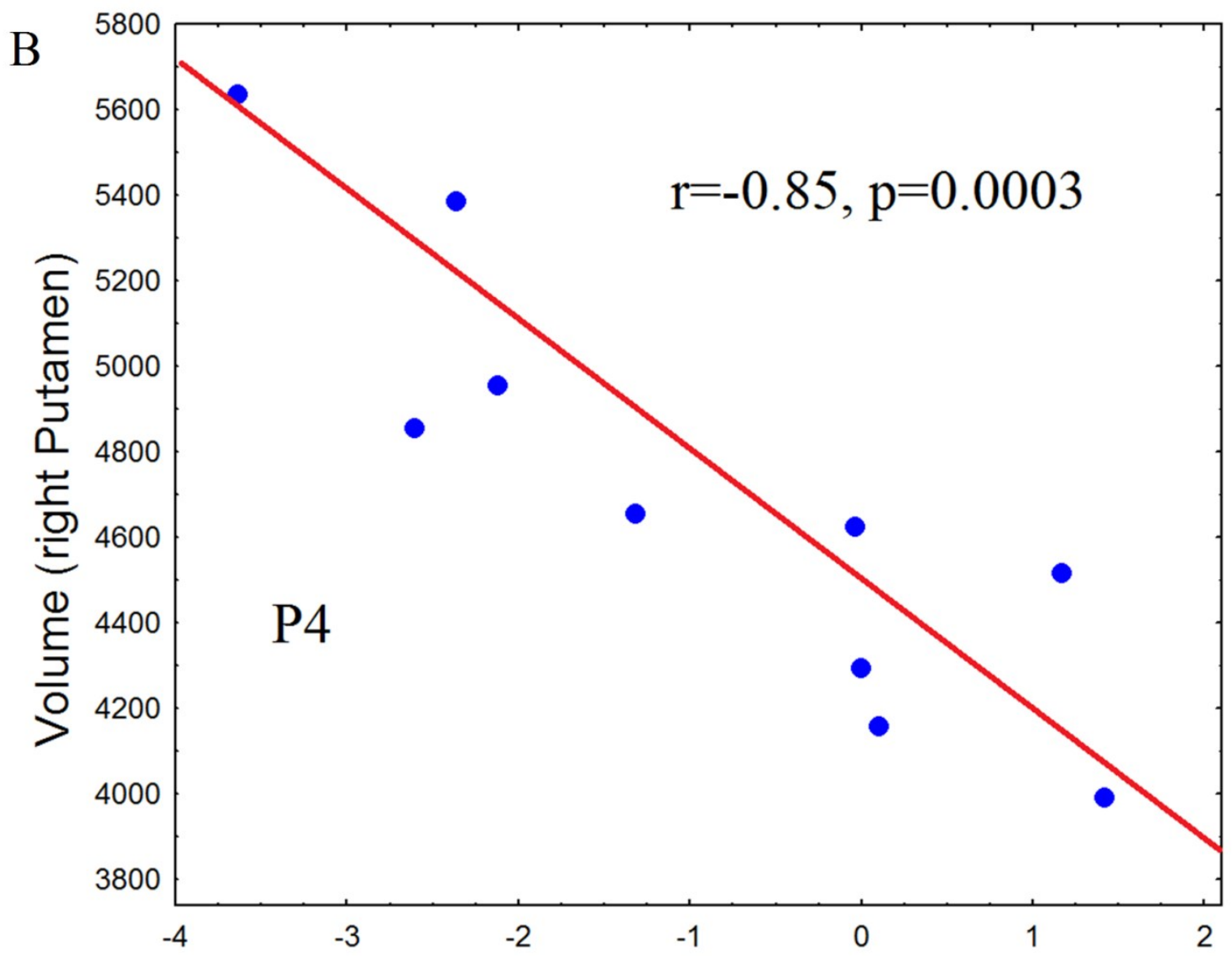

Figure 3. Results of clustering permutation's correlation analysis between the volume of brain structures and averaged a decrease of theta-rhythm power during tactile stimulation compared to rest. A: maps of significant correlations (black dots-electrodes showed significant correlations after Bonferroni correction); B: scatterplot depicted for the electrode marked by a white circle.

The correlation analysis between tactile EEG response and patients outcome showed that patients with better outcome (recovery) had a more pronounced decrease of the theta-rhythm PSD during tactile stimulation (trend significance: $r=-0.55, p=0.01$ ). The outcome for each patient was evaluated by the attending physician based on clinical signs. 


\section{EEG in response to tactile stimulation in a coma}

The increase of beta-rhythm power in the central areas correlated with the thickness of paracentral $(r=0.82, p=0.0005)$ and precentral areas bilaterally $(r=0.80, p=0.0007)$.
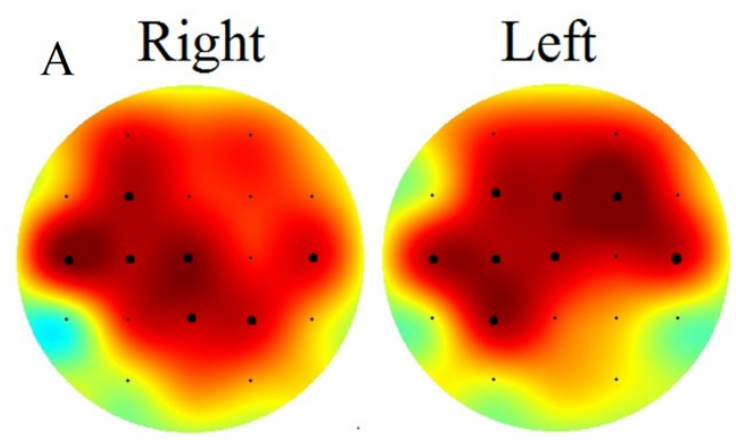

\section{Paracentral area}
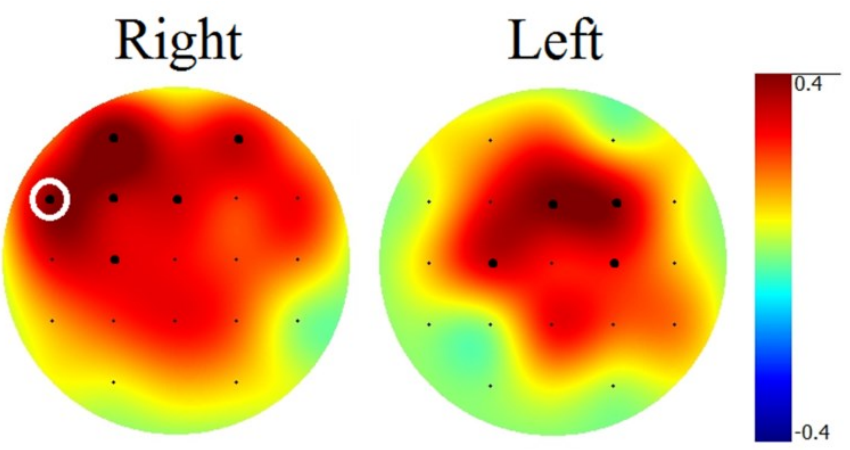

Precentral area

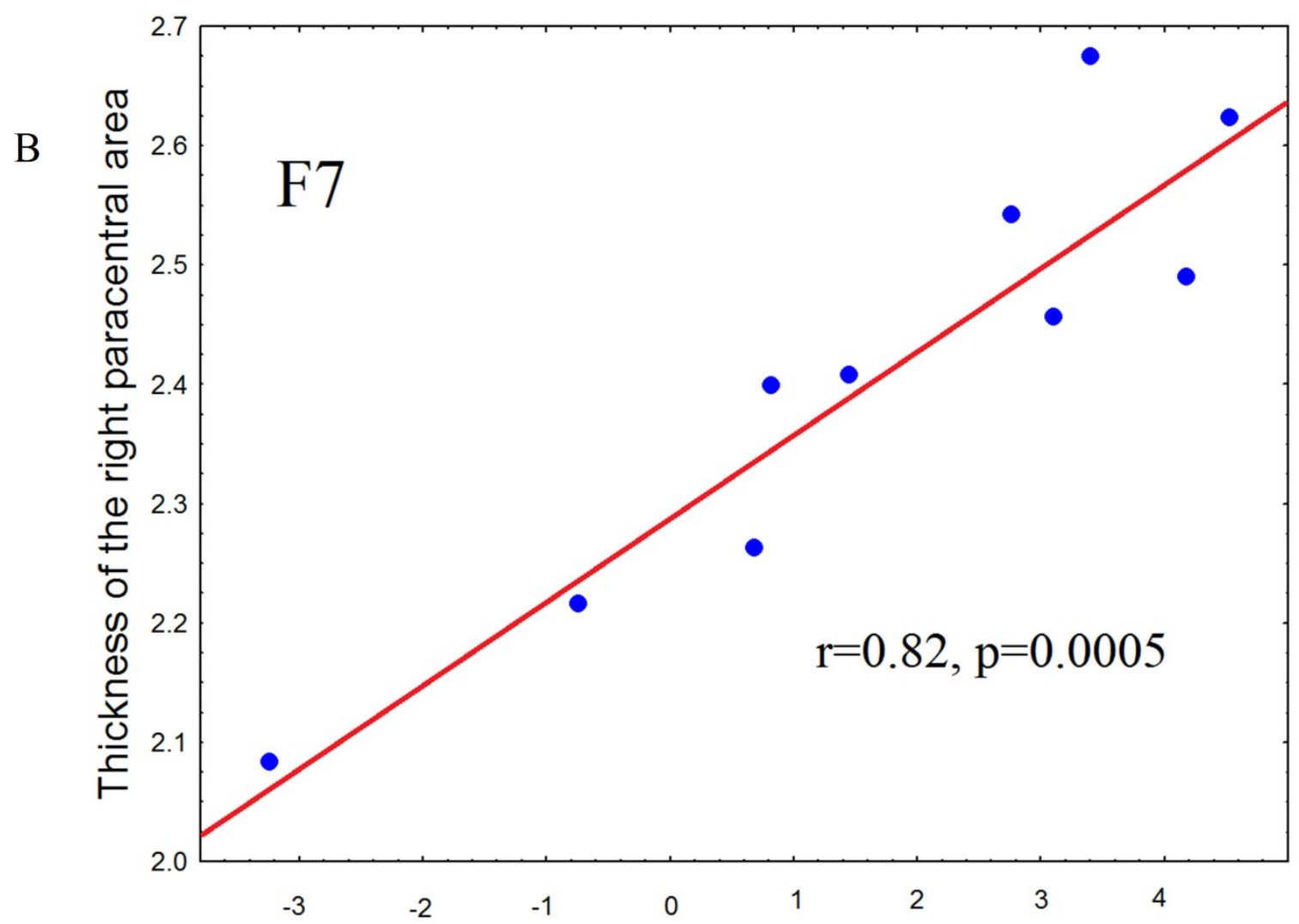

Figure 4. Results of correlation analysis between cortical thickness and averaged increase of beta-rhythm power during tactile stimulation compared to the rest. A: maps of significant correlations (black dots - electrodes showed significant correlations after Bonferroni correction); B: scatterplot depicted for the electrode marked by a white circle.

\section{Discussion}

Our findings indicated that the cortical thickness, volume of the cortex, and the volume of subcortical structures decreased in comatose patients compared to healthy subjects. Our results 


\section{EEG in response to tactile stimulation in a coma}

were also consistent with previous data showed that patients after severe brain injury exhibited significant atrophy across all examined regions, including regions known to be involved in the regulation of electrocortical arousal, sleep-wake rhythms, and conscious behavior [13]. Extensive atrophy was detected in globus pallidus, putamen, hippocampus, thalamus, the caudate nucleus, brainstem, and basal forebrain [19].

The cortical atrophy could be associated with traumatic reconstruction of the brain structure induced by injury and lead to impaired tactile perception. In particular, we found that the morphometry indices significantly correlated with EEG response during tactile stimulation in paracentral and precentral gyri only in comatose patients and could be critical for these processes. So, the higher was the cortical thickness in the central areas, the more pronounced was EEG response to tactile stimulation. Our findings indicated that the increase of the betarhythm PSD in response to tactile stimulation was correlated with the volume of variable cortical and sub-cortical thickness and thickness of paracentral and precentral areas associated with sensory-motor networks, but mostly with neural networks responsible for tactile perception $[47,48]$. The increase of beta-rhythm power during stimulation correlated with the thickness of the paracentral and precentral area. This response to tactile stimuli is well-known and could be associated with activity aimed at stimuli processing [49]. Paracentral area and postcentral area are the primary somatosensory cortex location, each hemisphere of which receives tactile and proprioceptive perception from the opposite side of the body in the manner of the inverted homunculus. The secondary somatosensory cortex is present in the vicinity of the lateral fissure, receives information from both sides of the body, and has multiple connections with the premotor cortex, insular cortex, amygdala, and hippocampus [50].

Simultaneously, the decrease of theta-rhythm power during tactile stimulation in comatose patients correlated with brain preservation from damage and was more prominent during unpleasant stimulation. Our results show (albeit at a trend level) that the higher decrease of theta-band PSD also was most pronounced in patients with a better outcome. Our findings indicated that specific for comatose patients respond induced by tactile stimulation and associated with brain regions related to consciousness. In particular, the global hallmark of impaired consciousness appears to be a multifaceted dysfunctional connectivity pattern with both within-network loss of connectivity in a widespread network of cortical and subcortical regions, including insula and putamen [51]. In our study, the localization of brain regions, correlated with the tactile EEG response of comatose patients, indicated that this response carried out by at least two main functional awareness networks. The external network, or executive control network, subserved by variable cortical regions and the internal awareness network, or default mode network (DMN), appears to be involved in self-related processes [52] and has been proposed as the locus of conscious awareness [53]. Previous studies also reported reduced connectivity in the DMN of patients in coma, vegetative state, and minimally conscious state, which correlated with the level of consciousness.

\section{Conclusion}

This study shows that EEG response to tactile stimulation correlates with brain damage after TBI and potentially predicts comma outcome. We found that the cortical thickness, as well as volume of the cortex and the volume of subcortical structures, decreased in comatose patients compared to healthy subjects. The specific tactile response (the increase of higher alpha and beta rhythms) found both in patients and healthy volunteers, correlated with the thickness of the somatosensory cortex. The nonspecific response to tactile stimulation (the decrease of spectral power in thetarange) was associated with the more preserved cortical and subcortical volume in the brain areas known as parts of awareness supporting networks. Patients with better outcome (recovery) had a 


\section{EEG in response to tactile stimulation in a coma}

more pronounced decrease of the theta-rhythm PSD during tactile stimulation. Our findings suggest that both specific and nonspecific oscillatory responses to tactile stimulation can indicate the severity of brain damage after TBI, predict coma outcome, and serve as biomarkers for comatose patients' rehabilitation.

\section{Funding}

The reported study was funded by RFBR according to the research project № 18-00-01670 (1800-01511) and the state assignment of Ministry of Education and Science of the Russian Federation for 2019-2021 (No. AAAA-A17-117092040004-0).

\section{Author Contributions}

G. Portnova: conceptualization, data curation, formal analysis, funding acquisition, investigation, methodology, project administration, resources, supervision, visualization, original draft, review \& editing.

D. Filatova: formal analysis, investigation, validation, visualization.review \& editing.

I. Girzhova: formal analysis, investigation, validation, visualization.

V. Podlepich: formal analysis, investigation, software, project administration

A. Tetereva: formal analysis, investigation

O. Martynova: conceptualization, supervision, visualization, review \& editing.

\section{References}

1. Lutkenhoff, E.S.; Chiang, J.; Tshibanda, L.; Kamau, E.; Kirsch, M.; Pickard, J.D.; Laureys, S.; Owen, A.M.; Monti, M.M. Thalamic and extrathalamic mechanisms of consciousness after severe brain injury. Ann Neurol 2015, 78, 68-76.

2. Lazarus, M.; Chen, J.F.; Urade, Y.; Huang, Z.L. Role of the basal ganglia in the control of sleep and wakefulness. Curr Opin Neurobiol 2013, 23, 780-785.

3. Lazarus, M.; Huang, Z.L.; Lu, J.; Urade, Y.; Chen, J.F. How do the basal ganglia regulate sleep-wake behavior? Trends Neurosci 2012, 35, 723-732.

4. Schiff, N.D. Central thalamic contributions to arousal regulation and neurological disorders of consciousness. Ann N Y Acad Sci 2008, 1129, 105-118.

5. Tomaiuolo, F.; Carlesimo, G.A.; di Paola, M.; Petrides, M.; Fera, F.; Bonanni, R.; Formisano, R.; Pasqualetti, P.; Caltagirone, C. Gross morphology and morphometric sequelae in the hippocampus, fornix, and corpus callosum of patients with severe nonmissile traumatic brain injury without macroscopically detectable lesions: a T1 weighted MRI study. J Neurol Neurosurg Psychiatry 2004, 75, 1314-1322.

6. Crick, F.; Koch, C. A framework for consciousness. Nat Neurosci 2003, 6, 119-126.

7. Dehaene, S.; Changeux, J.P. Experimental and theoretical approaches to conscious processing. Neuron 2011, 70, 200-227.

8. He, J.H.; Yang, Y.; Zhang, Y.; Qiu, S.Y.; Zhou, Z.Y.; Dang, Y.Y.; Dai, Y.W.; Liu, Y.J.; $\mathrm{Xu}, \mathrm{R} . \mathrm{X}$. Hyperactive external awareness against hypoactive internal awareness in disorders of consciousness using resting-state functional MRI: highlighting the involvement of visuo-motor modulation. NMR Biomed 2014, 27, 880-886. 


\section{EEG in response to tactile stimulation in a coma}

9. Hannawi, Y.; Lindquist, M.A.; Caffo, B.S.; Sair, H.I.; Stevens, R.D. Resting brain activity in disorders of consciousness: a systematic review and meta-analysis. Neurology 2015, 84, 1272-1280.

10. Laureys, S.; Goldman, S.; Phillips, C.; van Bogaert, P.; Aerts, J.; Luxen, A.; Franck, G.; Maquet, P. Impaired effective cortical connectivity in vegetative state: preliminary investigation using PET. Neuroimage 1999, 9, 377-382.

11. Jang, S.; Kwak S. The upper ascending reticular activating system between intralaminar thalamic nuclei and cerebral cortex in the human brain. J Kor Phys Ther 2017, 29, 109114.

12. Laureys, S. The neural correlate of (un)awareness: lessons from the vegetative state. Trends Cogn Sci 2005, 9, 556-559.

13. Laureys, S.; Faymonville, M.E.; Luxen, A.; Lamy, M.; Franck, G.; Maquet, P. Restoration of thalamocortical connectivity after recovery from persistent vegetative state. Lancet 2003, 355, 1790-1791.

14. Mecarelli, O. B.; Brienza, M.; Grippo, A.; Amantini, A. Disorders of Consciousness. In Clinical Electroencephalography, 1st ed.; Mecarelli, O. B., Eds.; Springer: Cham, Switzerland, 2019; pp. 731-765, ISBN 978-3-030-04573-9.

15. Emidio, A.C.; Faria, R.; Patricio, P.; Canas, N.; Messias, A.; Meneses-Oliveira, C. Spindle Coma in the Intensive Care Unit: Different Aetiologies-Different Outcomes. Eur J Case Rep Intern Med 2019, 6.

16. Ardeshna, N. I. EEG and Coma. The Neurodiagnostic Journal 2016, 56, 1-16.

17. Tshibanda, L.; Vanhaudenhuyse, A.; Galanaud, D.; Boly, M.; Laureys, S.; Puybasset, L. Magnetic resonance spectroscopy and diffusion tensor imaging in coma survivors: promises and pitfalls. Prog Brain Res 2009, 177, 215-229.

18. Potapov, A.A.; Zakharova, N.E.; Kornienko, V.N.; Pronin, I.N.; Alexandrova, E.V.; Zaitsev, O.S.; Likhterman, L.B.; Gavrilov, A.G.; Danilov, G.V.; Oshorov, A.V. et al. Neuroanatomical basis for traumatic coma: clinical and magnetic resonance correlates. Zh Vopr Neirokhir Im N N Burdenko 2014, 78, 4-13.

19. Firsching, R.; Woischneck, D.; Klein, S.; Reissberg, S.; Döhring, W.; Peters, B. Classification of severe head injury based on magnetic resonance imaging. Acta Neurochir (Wien) 2001, 143, 263-271.

20. Brenner, R.P. The interpretation of the EEG in stupor and coma. The neurologist 2005, 11, 271-284.

21. Husain, A.M. Electroencephalographic assessment of coma. J Clin Neurophysiol 2006, 23, 208-220.

22. Weiss, N.; Galanaud, D.; Carpentier, A.; Tezenas de Montcel S.; Naccache, L.; Coriat, P.; Puybasset, L. A combined clinical and MRI approach for outcome assessment of traumatic head injured comatose patients. J Neurol 2008, 255, 217-223.

23. Bekinschtein, T.; Gleichgerrcht, E.; Manes, F. Acute loss of consciousness. In Handbook of clinical neurology, 3rd ed.; Grafman, J., Salazar, A.M., Eds.; Elsevier: Amsterdam, Netherlands, 2015; Volume 127, pp. 197-204, ISBN 0720472008.

24. Abraira, V.E.; Ginty, D.D. The sensory neurons of touch. Neuron 2013, 79, 618-39.

25. Vallbo, A.O. A system of unmyelinated afferents for innocuous mechanoreception in the human skin. Brain Res 1993, 628, 301-4. 


\section{EEG in response to tactile stimulation in a coma}

26. Olausson, H.L. Unmyelinated tactile afferents signal touch and project to insular cortex. Nat Neurosci 2002, 5, 900-4.

27. Björnsdotter, M.L. Somatotopic organization of gentle touch processing in the posterior insular cortex. J Neurosci 2009, 29, 9314-20.

28. Goodwin, A.W. Physiological responses of sensory afferents in glabrous and hairy skin of humans and monkeys. The Senses: A Compr Ref 2010, 6, 39-54.

29. Morrison, I. CT afferents. Curr Biol 2010, $C B$ 22, R77-R78.

30. I. Gordon, A.V. Brain mechanisms for revealing affective touch. Hum Brain Mapp, 2011, 34(4), 914-22.

31. Ackerley, R.; Eriksson, E.; Wessberg, J. Ultra-late EEG potential evoked by preferential activation of unmyelinated tactile afferents in human hairy skin. Neurosci letters $\mathbf{2 0 1 3}$, $535,62-66$.

32. André-Obadia, N. Z. Recommendations for the use of electroencephalography and evoked potentials in comatose patients. Neurophysiologie clinique 2018, 48(3), 143-69.

33. Fischl, B.; Salat, D.H. Whole brain segmentation: automated labeling of neuroanatomical structures in the human brain. Neuron 2002, 33, 341-355.

34. Fischl, B.; Salat, D.H. Sequence-independent segmentation of magnetic resonance images. Neuroimage 2004a, 23 Suppl 1, S69-84.

35. Sled, J.G.; Zijdenbos, A.P. A nonparametric method for automatic correction of intensity nonuniformity in MRI data. IEEE Trans Med Imaging 1998, 17, 87-97.

36. Fischl, B.; Liu, A. Automated manifold surgery: constructing geometrically accurate and topologically correct models of the human cerebral cortex. IEEE Trans Med Imaging 2001, 20, 70-80.

37. Segonne, F.; Pacheco, J.; Fischl, B. Geometrically accurate topology-correction of cortical surfaces using nonseparating loops. IEEE Trans Med Imaging 2007, 26, 518-529.

38. Dale, A.M.; Sereno, M.I. Improved localization of cortical activity by combining EEG and MEG with MRI cortical surface reconstruction: a linear approach. $J$ Cogn Neurosci 1993, 5, 162-176.

39. Dale, A.M.; Fischl, B.; Sereno, M.I. Cortical surface-based analysis. I. Segmentation and surface reconstruction. Neuroimage 1999, 9, 179-194.

40. Fischl, B.; Dale, A.M. Measuring the thickness of the human cerebral cortex from magnetic resonance images. Proc Natl Acad Sci U S A 2000, 97, 11050-11055.

41. Fischl, B.; Sereno, M.I.; Dale, A.M. Cortical surface-based analysis. II: Inflation, flattening, and a surface-based coordinate system. Neuroimage 1999a, 9, 195-207.

42. Fischl, B.; Sereno, M.I.; Tootell, R.B.; Dale, A.M. High-resolution intersubject averaging and a coordinate system for the cortical surface. Hum Brain Mapp 1999b, 8, 272-284.

43. Fischl, B.; van der Kouwe, A.; Destrieux, C.; Halgren, E.; Segonne, F. Automatically parcellating the human cerebral cortex. Cereb Cortex 2004, 14, 11-22.

44. Desikan, R.S.; Segonne, F.; Fischl, B.; Quinn, B.T.; Dickerson, B.C. An automated labeling system for subdividing the human cerebral cortex on MRI scans into gyral based regions of interest. Neuroimage 2006, 31, 968-980.

45. Higuchi, T. Approach to an irregular time series on the basis of the fractal theory. Physica D 1988, 31, 277-283. 


\section{EEG in response to tactile stimulation in a coma}

46. Ktonas, P.Y.; Papp, N. Instantaneous envelope and phase extraction from real signals: theory, implementation, and an application to EEG analysis. Signal Processing 1980, 2 , 373-385.

47. Kitada, R.; Doizaki, R.; Kwon, J.; Tanigawa, T. Brain networks underlying tactile softness perception: A functional magnetic resonance imaging study. NeuroImage 2019, 197, 156-166.

48. Qi, H.-X.P. Somatosensory areas of the cerebral cortex: architectonic characteristics and modular organization, $1^{\text {st }}$ ed. ; Academic Press: NY ; 2008.

49. Pfurtscheller, G.; Lopes da Silva, F.H. Event-related EEG/MEG synchronization and desynchronization: Basic principles. Clinical Neurophysiology 1999, 110 (11), 1842-57.

50. Jeffry, J.; Kim, S.; Chen, Z.F. Itch signaling in the nervous system. Physiology (Bethesda) 2011, 26 (286).

51. Di Perri, C.; Stender, J.; Laureys, S.; Gosseries, O. Functional neuroanatomy of disorders of consciousness. Epilepsy \& Behavior 2014, 30, 28-32.

52. Vanhaudenhuyse, A.; Demertzi, A.; Schabus, M. Two distinct neuronal networks mediate the awareness of environment and of self. J Cogn Neurosci 2011, 23, 570-8.

53. Vanhaudenhuyse, A.; Noirhomme, Q.; Tshibanda, L.J. Bruno M.A. Default network connectivity reflects the level of consciousness in noncommunicative brain-damaged patients. Brain 2010, 133 (Pt 1), 161-71. 
EEG in response to tactile stimulation in a coma

Table 1. Clinical information about patients.

\begin{tabular}{|c|c|c|c|c|c|c|}
\hline Patient & Cause of trauma & Age & $\begin{array}{l}\text { Time from } \\
\text { accident till } \\
\text { EEG } \\
\text { recording }\end{array}$ & $\begin{array}{l}\text { Coma } \\
\text { Glazgo } \\
\text { Scale }\end{array}$ & $\begin{array}{l}\text { MRI results } \\
\text { (regions of brain } \\
\text { contusions) }\end{array}$ & $\begin{array}{c}\text { Outcome ( } \\
\text { Glasgow } \\
\text { Outcome Scale } \\
\text { extended) }\end{array}$ \\
\hline 1 & car accident & 18 & 7 days & 5 & $\begin{array}{l}\text { right frontal and } \\
\text { the corpus } \\
\text { callosum }\end{array}$ & 6 \\
\hline 2 & car accident & 41 & 28 days & 7 & $\begin{array}{l}\text { right temporal- } \\
\text { frontal region } \\
\text { left temporal- } \\
\text { frontal region, } \\
\text { right hemisphere } \\
\text { of the cerebellum }\end{array}$ & 3 \\
\hline 3 & car accident & 43 & 12 days & 7 & $\begin{array}{l}\text { front temporal } \\
\text { lobes of the right } \\
\text { hemisphere } \\
\text { left temporal and } \\
\text { occipital lobes }\end{array}$ & 5 \\
\hline 4 & car accident & 26 & $\begin{array}{c}2 \text { months } 2 \\
\text { days }\end{array}$ & 6 & $\begin{array}{c}\text { left frontal and } \\
\text { right occipital } \\
\text { lobe. }\end{array}$ & 3 \\
\hline 5 & beating & 32 & $\begin{array}{l}1 \text { month } 18 \\
\text { days }\end{array}$ & 5 & $\begin{array}{c}\text { brain stem } \\
\text { left basal temporal } \\
\text { region. }\end{array}$ & 3 \\
\hline 6 & fall from a height & 51 & 7 days & 6 & $\begin{array}{c}\text { left frontal and } \\
\text { temporal regions, } \\
\text { diffuse axonal } \\
\text { damage }\end{array}$ & 5 \\
\hline 7 & car accident & 34 & $\begin{array}{c}2 \text { months } 25 \\
\text { days }\end{array}$ & 7 & $\begin{array}{l}\text { diffuse axonal } \\
\text { damage }\end{array}$ & 6 \\
\hline 8 & car accident & 34 & 4 days & 5 & $\begin{array}{l}\text { the corpus } \\
\text { callosum, } \\
\text { diffuse axonal } \\
\text { damage }\end{array}$ & 3 \\
\hline 9 & car accident & 38 & 8 days & 6 & brain stem & 5 \\
\hline 10 & car accident & 26 & 4 days & 5 & $\begin{array}{l}\text { the corpus } \\
\text { callosum, } \\
\text { diffuse axonal } \\
\text { damage }\end{array}$ & 7 \\
\hline
\end{tabular}




\section{EEG in response to tactile stimulation in a coma}

Table 2. Cortical thickness in areas associated with tactile stimulation and level of consciousness (according to literature data) and their cross-group differences

\begin{tabular}{|c|c|c|c|c|c|}
\hline & Area & $\begin{array}{l}\text { Thickness in Control } \\
\text { group (mean } \pm \text { std) }\end{array}$ & $\begin{array}{l}\text { Thickness in } \\
\text { Comatose patients } \\
(\text { mean } \pm \text { std })\end{array}$ & $\begin{array}{l}\text { Z (Mann-Whitney } \\
\text { U Test) }\end{array}$ & p-level \\
\hline \multirow[b]{13}{*}{ Right } & Anterior cingulate & $2.7 \pm 0.3$ & $2.5 \pm 0.2$ & 1.36 & 0.17 \\
\hline & Posterior cingulate & $2.5 \pm 0.2$ & $2.5 \pm 0.2$ & 1.16 & 0.24 \\
\hline & Middle-frontal & $2.6 \pm 0.1$ & $2.6 \pm 0.2$ & 0.78 & 0.44 \\
\hline & Parahippocampal & $2.8 \pm 0.3$ & $2.8 \pm 0.3$ & -1.14 & 0.26 \\
\hline & Inferior-parietal & $2.5 \pm 0.1$ & $2.4 \pm 0.1$ & 1.83 & 0.07 \\
\hline & Superior parietal & $2.2 \pm 0.1$ & $2.2 \pm 0.1$ & -0.08 & 0.93 \\
\hline & Paracentral & $2.5 \pm 0.1$ & $2.3 \pm 0.1$ & 2.22 & 0.03 \\
\hline & Postcentral & $2.0 \pm 0.1$ & $2.0 \pm 0.1$ & 1.00 & 0.32 \\
\hline & Precentral & $2.6 \pm 0.1$ & $2.3 \pm 0.2$ & 3.00 & 0.00 \\
\hline & Precuneus & $2.4 \pm 0.1$ & $2.4 \pm 0.1$ & -1.55 & 0.12 \\
\hline & Supramarginal & $2.6 \pm 0.1$ & $2.4 \pm 0.2$ & 2.77 & 0.01 \\
\hline & Insula & $3.1 \pm 0.1$ & $2.9 \pm 0.2$ & 3.16 & 0.00 \\
\hline & Mean Thickness & $2.6 \pm 0.1$ & $2.1 \pm 0.1$ & 3.54 & 0.00 \\
\hline \multirow[b]{13}{*}{ Left } & Anterior cingulate & $2.8 \pm 0.2$ & $2.7 \pm 0.4$ & 1.11 & 0.27 \\
\hline & Posterior cingulate & $2.6 \pm 0.1$ & $2.4 \pm 0.2$ & 1.94 & 0.05 \\
\hline & Middle-frontal & $2.5 \pm 0.1$ & $2.4 \pm 0.2$ & 0.89 & 0.37 \\
\hline & Parahippocampal & $2.8 \pm 0.3$ & $2.9 \pm 0.2$ & -0.83 & 0.41 \\
\hline & Inferior-parietal & $2.5 \pm 0.1$ & $2.4 \pm 0.1$ & 1.22 & 0.22 \\
\hline & Superior parietal & $2.2 \pm 0.1$ & $2.1 \pm 0.1$ & 1.58 & 0.11 \\
\hline & Paracentral & $2.4 \pm 0.2$ & $2.3 \pm 0.1$ & 1.55 & 0.12 \\
\hline & Postcentral & $2.0 \pm 0.1$ & $2.0 \pm 0.1$ & 1.05 & 0.29 \\
\hline & Precentral & $2.6 \pm 0.1$ & $2.3 \pm 0.2$ & 3.36 & 0.00 \\
\hline & Precuneus & $2.4 \pm 0.1$ & $2.4 \pm 0.1$ & 0.64 & 0.52 \\
\hline & Supramarginal & $2.6 \pm 0.1$ & $2.4 \pm 0.2$ & 2.58 & 0.01 \\
\hline & Insula & $3.1 \pm 0.1$ & $2.8 \pm 0.2$ & 2.55 & 0.01 \\
\hline & Mean Thickness & $2.6 \pm 0.1$ & $2.4 \pm 0.1$ & 3.05 & 0.00 \\
\hline
\end{tabular}


Table 3. The volume of brain structures associated with tactile stimulation and the level of consciousness (according to the literature) and their intergroup differences

\begin{tabular}{|c|c|c|c|c|c|}
\hline & Area & $\begin{array}{l}\text { Volume in Control group } \\
\qquad(\text { mean } \pm \text { std })\end{array}$ & $\begin{array}{l}\text { Volume in Comatose } \\
\text { patients (mean } \pm \text { std) }\end{array}$ & $\begin{array}{l}\text { Z (Mann- } \\
\text { Whitney } \\
\text { U Test) }\end{array}$ & p-level \\
\hline \multirow[b]{8}{*}{ Right } & Cerebellum Cortex & $60694.6 \pm 8020.7$ & $53845.4 \pm 6696.6$ & 2.00 & 0,05 \\
\hline & Thalamus Proper & $7921.3 \pm 954.7$ & $6698.6 \pm 1125.7$ & 2.61 & 0,01 \\
\hline & Caudate & $3651.2 \pm 456.0$ & $3563.7 \pm 490.4$ & 0.55 & 0,58 \\
\hline & Putamen & $4983.8 \pm 564.9$ & $4459.5 \pm 654.7$ & 2.00 & 0,05 \\
\hline & Pallidum & $2022.6 \pm 275.2$ & $1633.0 \pm 322.9$ & 2.83 & 0,00 \\
\hline & Brain Stem & $21693.8 \pm 3270.3$ & $20388.7 \pm 3502.0$ & 1.33 & 0,18 \\
\hline & Hippocampus & $4206.7 \pm 538.4$ & $3962.8 \pm 536.1$ & 0.89 & 0,37 \\
\hline & Amygdala & $1731.2 \pm 241.1$ & $1840.2 \pm 350.3$ & -0.72 & 0,47 \\
\hline \multirow[b]{8}{*}{ Left } & Cerebellum Cortex & $58892.6 \pm 7735.6$ & $52447.6 \pm 6402.8$ & 2.16 & 0,03 \\
\hline & Thalamus Proper & $7930.5 \pm 669.9$ & $7129.0 \pm 1031.5$ & 1.83 & 0,07 \\
\hline & Caudate & $3661.9 \pm 516.2$ & $3305.2 \pm 576.0$ & 1.89 & 0,06 \\
\hline & Putamen & $4796.9 \pm 644.4$ & $4927.6 \pm 911.8$ & -0.17 & 0,87 \\
\hline & Pallidum & $2068.5 \pm 256.5$ & $1814.8 \pm 317.9$ & 1.89 & 0,06 \\
\hline & Brain Stem & $21773.0 \pm 3270.3$ & $20401.7 \pm 3176.0$ & 1.34 & 0,19 \\
\hline & Hippocampus & $4025.6 \pm 431.9$ & $3948.8 \pm 589.4$ & 0.55 & 0,58 \\
\hline & Amygdala & $1533.2 \pm 214.3$ & $1688.9 \pm 346.7$ & -1.28 & 0,20 \\
\hline \multicolumn{2}{|r|}{ Cortex Volume } & $503393,8 \pm 55903,0$ & $462949.0 \pm 46353.0$ & 1.28 & 0.20 \\
\hline \multicolumn{2}{|r|}{ Surface Holes } & $46,5 \pm 15,0$ & $207,6 \pm 91,6$ & $-4,16$ & 0,00 \\
\hline
\end{tabular}


